\title{
Does Peripheral Lower Body Blood Flow Restriction Training Effect Aerobic Adaptations in Recreationally Active Adults? A Case Series
}

Research Brief

\begin{abstract}
Austin Bennett ${ }^{1}$, Sean Collins ${ }^{1}$, Kaitlyn King ${ }^{1}$, Caitlyn Harper ${ }^{1}$, Jill Lucas ${ }^{1}$, Jeffrey Herrick ${ }^{1}$
${ }^{1}$ Department of Exercise Physiology, University of Lynchburg, Lynchburg, VA

Open Access

Published: January 5, 2022

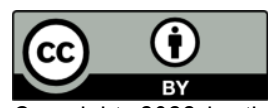

Copyright, 2022 by the authors. Published by Pinnacle Science and the work is licensed under the Creative Commons Attribution 4.0 International License. To view a copy of this license, visit http://creativecommons .org/licenses/by/4.0/

Abstract

Introduction: Blood flow restriction (BFR) training is a novel training method that has been shown to promote positive aerobic and anaerobic adaptations under low intensity exercise by inhibiting blood flow to target tissue resulting in hypoxia and metabolic byproduct accumulation. This has been shown to have a direct positive effect on aerobic performance adaptation. The purpose was to explore the effect of BFR training on aerobic performance.

Methods: Seven recreationally active adults were randomly assigned to either the BFR group $(n=4, B F R)$ or non-BFR group $(n=3, C O N)$. Three testing sessions were conducted throughout the study (Pre-Test, Mid-Test, Post-Test) which consisted of a graded cycle ergometer maximum oxygen consumption $\left(\mathrm{VO}_{2 \max }\right)$ test using COSMED-K5 indirect calorimetry. Eleven BFR training sessions were performed consisting of 20 -min of cycling at $35-45 \%$ of heart rate reserve (HRR) with at $60 \%(\mathrm{BFR})$ or $5 \%(\mathrm{CON})$ arterial occlusion pressure (AOP) on the BFR cuffs.

Results: Absolute $\mathrm{VO}_{2 \max }$, Relative $\mathrm{VO}_{2 \max }$, respiratory exchange ratio (RER), maximum heart rate $\left(\mathrm{HR}_{\max }\right)$, and maximum rate of perceived exertion ( $\left.\mathrm{RPE}_{\max }\right)$ reported no significant difference between BFR and CON. There was a significant difference $(p<0.05)$ found in time to reach $\mathrm{VO}_{2 \max }$ and maximum watts $\left(\mathrm{W}_{\max }\right)$ reached which declined over the course of the training intervention.

Conclusions: Bilateral lower limb aerobic BFR training resulted in no change in $\mathrm{VO}_{2 \max }$ over seven weeks.
\end{abstract}

Journal of Exercise and Nutrition: 2022, Volume 5 (Issue 1): 2

ISSN: $2640-2572$
Key Words: BFR, $\mathrm{VO}_{2 \max }$, Recumbent bike, Occlusion, cycle training.

Corresponding author: Sean Collins, collins.s@lynchburg.edu

\section{Introduction}

Blood flow restriction (BFR) training is a novel training method that involves restricting limb arterial blood flow and venous return promoting localized

hypoxia ${ }^{9}$. Additionally, BFR requires performing a large number of repetitions ( $\sim 75$ total reps) at relatively low volume $(20-30 \% \text { of one-repetition maximum }(1 \mathrm{RM}))^{9}$. The combination of these two training features leads to a buildup of metabolic waste products that may lead to accelerated cellular adaptations ${ }^{10}$. As a result, BFR has been implemented in a wide range of athletic populations, such as resistance training, interval training, and aerobic training ${ }^{8}$. 
Blood flow restriction has been demonstrated to be effective at increasing muscular strength and hypertrophy in a wide range of power athletes ${ }^{1}$. Due to the increased rate of muscular and aerobic adaptations observed during BFR training, and its safe application, BFR presents itself as a possible approach to increase muscular performance in non-athletic populations. Specifically, BFR has shown positive physiological adaptations when paired with treadmill walking and jogging, as well as with stationary cycling in mixed populations ${ }^{5,6,8}$. Both aerobic and anaerobic muscular adaptations have been seen during walking or cycling for five to 20 minutes with an intensity of $<50 \% \mathrm{VO}_{2 \text { max }}$ or heart rate reserve (HRR), two to three times per week for more than three weeks ${ }^{9}$. Therefore, the purpose of this study is to evaluate the effects of peripheral blood flow restriction of bilateral lower limbs during aerobic stationary recumbent cycle training on $\mathrm{VO}_{2 \max }$ and other ventilatory adaptations while training twice a week for seven weeks.

\section{Scientific Methods}

\section{Participants}

Seven participants volunteered for the study. Participants where pre-screened for readiness via the PARQ + and American College of Sports Medicine's pre-participation screening algorithm 3,11 . Prior to any testing or training, participants were randomly assigned to one of two groups; treatment group (BRF) ( $\mathrm{n}=4$, BFR training- $60 \%$ AOP) or the control group (CON) ( $\mathrm{n}=3$, non-BFR training-5\% AOP), using a single-blind method (Table 1). The study was approved by the Institutional Review Board.

Table 1. Anthropometric data

\begin{tabular}{lll}
\hline & Treatment $(\mathbf{n}=\mathbf{4})$ & Control $(\mathbf{n}=\mathbf{3})$ \\
Age (years) & $30.00 \pm 5.29$ & $20.67 \pm 2.08$ \\
Weight $(\mathrm{kg})$ & $87.74 \pm 13.70$ & $83.90 \pm 15.09$ \\
Height $(\mathrm{m})$ & $1.73 \pm 0.09$ & $1.70 \pm 0.10$ \\
Body Fat $(\%)$ & $23.54 \pm 12.97$ & $24.30 \pm 15.35$ \\
\hline
\end{tabular}

Protocol

Testing was performed three times throughout the duration of the seven-week study: at the beginning of the study (Pre-Test), after 5 training sessions (Mid-Test), and at the completion of seven weeks (Post-Test) (Figure 1). Anthropometrics were gathered at the beginning of each testing session to include: height (SECA 222 stadiometer, Chino, CA), weight (Tanita BWB-800AS; Arlington Heights, IL), resting heart rate (HR; Polar T31 heart rate monitor, Bethpage, NY) and resting blood pressure (BP) (OMRON HEM-712C; Kyoto, Japan). Body composition was measured using bioelectrical impedance analysis (BIA; Tanita BWB-800AS; Arlington Heights, IL). Arterial occlusion pressure (AOP) was obtained while each subject lay supine on a padded treatment table. A BFR cuff (Smart Cuff, Strongsville, OH) was fitted and placed around the left leg at the most proximal portion of the thigh. A doppler (Smart Cuff, Strongsville, $\mathrm{OH}$ ) was used to monitor peroneal artery blood flow and the cuff was inflated with a hand gauge (Smart Cuff, Strongsville, $\mathrm{OH}$ ) until the peroneal pulse was no longer audible, and the number was recorded as the AOP.

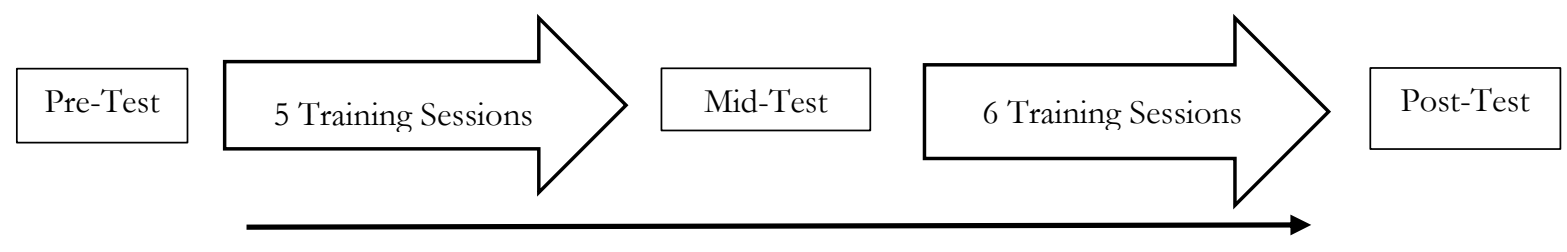

Figure 1. Timeline of testing events.

7 Weeks

Each subject was then fitted appropriately for the COSMED K5 (Rome, Italy) portable indirect calorimeter (Concord, CA) before performing a graded exercise cycle ergometer test on a Lode stationary bike (Groningen, Netherlands) to determine $\mathrm{VO}_{2 \max }$ (criteria: a $\mathrm{HR}$ greater than $95 \%$ of age-predicted max, respiratory exchange ratio $($ RER $)>1.1$, and rate of perceived exertion $($ RPE $)>18$ ). The graded exercise protocol consisted of cycling at 60 $\mathrm{rpm}$ with a starting workload of $100 \mathrm{~W}$ and $25 \mathrm{~W}$ increases every two minutes until volitional exhaustion. At the end of every two-minute stage, rate of perceived exertion (RPE) was recorded on a Borg 6-20 Scale ${ }^{4}$. Upon termination of the test maximum relative and absolute $\mathrm{VO}_{2 \max }$, RER, and a final RPE were recorded. 
Training Protocol

Participants performed eleven training sessions over seven weeks where they were fitted for a Polar HR monitor before beginning a five-minute warm-up on the recumbent bike (SportsArtfitness XT20 Mukilteo, WA or True Es700 O' Fallon, MO). The HR monitor was used to control the intensity for each training session at 35-45\% of their heart rate reserve (HRR). After the warm-up, BFR cuffs were placed bilaterally on the proximal thigh bilaterally and inflated to the designated percentage of AOP. The participants were instructed to pedal for 20 min at a pace equal to $35-45 \%$ HRR. Every five-minutes, RPE was recorded.

Statistical Analysis

Statistical analysis was conducted using eight 2x3 Repeated Measures ANOVA for Groups (treatment vs control) and Tests (Pre-, Mid-, and Post-) for AOP percent, absolute $\mathrm{VO}_{2 \max }$, relative $\mathrm{VO}_{2 \max }$, maximum $\mathrm{RER}\left(\mathrm{RER}_{\max }\right)$, maximum heart rate $\left(\mathrm{HR}_{\max }\right)$, maximum $\mathrm{RPE}\left(\mathrm{RPE} \mathrm{m}_{\max }\right)$, Time to reach $\mathrm{VO}_{2 \max }$, and maximum Watts $\left(\mathrm{W}_{\max }\right)$. The statistical analysis software used was JASP 10.2 (Amsterdam, Netherland). A Bonferroni post-hoc analysis was utilized for significant findings. For all analyses, the alpha level was set to 0.05 a priori.

\section{Results}

All enrolled subject $(\mathrm{N}=7)$ completed all training and testing sessions of the experimental design. Baseline performance data is presented in Table 2 . There were no significant differences $\left(F_{2,10}=0.87, p>0.05\right)$ in the AOP between groups (control v. treatment). As expected, there was a significant difference $\left(\mathrm{F}_{1,5}=742.37, p<0.05\right.$, $\left.\eta^{2}=0.98\right)$ between the BFR pressures during training with the cuff pressure for the BFR $(115.92 \pm 8.03 \mathrm{mmHg}) \mathrm{group}$ was significantly higher than the pressure for the CON $(9.06 \pm 1.13 \mathrm{mmHg})$.

Table 2. Baseline Performance Data*

\begin{tabular}{|c|c|c|}
\hline & $\begin{array}{l}\text { BFR } \\
(n=5)\end{array}$ & $\begin{array}{l}\text { Control } \\
(\mathrm{n}=3)\end{array}$ \\
\hline Absolute $\mathbf{V O}_{2 \max }\left(\mathrm{L} \cdot \mathrm{min}^{-1}\right)$ & $3.49 \pm 0.98$ & $3.99 \pm 0.52$ \\
\hline Relative $\mathbf{V O}_{2 \max }\left(\mathrm{ml} \cdot \mathrm{kg}^{-1} \cdot \mathrm{min}^{-1}\right)$ & $40.34 \pm 10.69$ & $48.67 \pm 12.20$ \\
\hline $\mathbf{H R}_{\max }(\mathrm{bpm})$ & $185.40 \pm 10.14$ & $194.33 \pm 1.16$ \\
\hline $\mathbf{R E R}_{\max }$ & $1.15 \pm 0.13$ & $1.08 \pm 0.06$ \\
\hline $\mathbf{R P E}_{\max }$ & $17.60 \pm 2.07$ & $17.33 \pm 3.06$ \\
\hline Time to Reach $\mathbf{V O}_{2 \max }(\mathrm{sec})$ & $613.80 \pm 194.09$ & $814.33 \pm 225.09$ \\
\hline $\mathbf{W}_{\max }(\mathrm{W})$ & $225.00 \pm 46.77$ & $258.33 \pm 38.19$ \\
\hline
\end{tabular}

*The was no significant differences $(p>0.05)$ between the physiological variables tested at baseline between groups

Following the training sessions, there were no significant interactions between the Groups and Tests for absolute $\mathrm{VO}_{2 \max }\left(\mathrm{F}_{2,10}=1.97, p=0.19, \eta^{2}=0.05\right)$, relative $\mathrm{VO}_{2 \max }\left(\mathrm{F}_{2,10}=1.50, p=0.27, \eta^{2}=0.04\right), \mathrm{HR}_{\max }\left(\mathrm{F}_{2,10}=0.13, \mathrm{p}=0.88\right.$, $\left.\eta^{2}=0.00\right), \mathrm{RER}_{\max }\left(\mathrm{F}_{2,10}=2.11, p=0.17, \eta^{2}=0.13\right)$, and $\mathrm{RPE}_{\max }\left(\mathrm{F}_{2,10}=0.92, p=0.43, \eta^{2}=0.07\right)$. Additionally, there were no significant main effects for Tests (pre-, mid-, post-) nor for Groups for the variables.

There were no significant interactions $\left(\mathrm{F}_{2,10}=3.38, p=0.8, \eta^{2}=0.03\right)$ between tests and groups for time to reach $\mathrm{VO}_{2 \max }$. Additionally, there was no significant difference between groups $\left(\mathrm{F}_{1,5}=0.23, p=0.66, \eta^{2}=0.04\right)$, but there was a significant difference between tests $\left(\mathrm{F}_{2,10}=4.21, p=0.047, \eta^{2}=0.04\right)$. The Pre-Test $(753.29 \pm 160.5 \mathrm{sec})$ was higher than Mid-Test $(708.75 \pm 146.63 \mathrm{sec})$ and higher than Post-Test $(688.09 \pm 151.47 \mathrm{sec})$. There was no significant difference between the Mid-test (708.75 $\pm 146.63 \mathrm{sec})$ and the Post-test $(688.09 \pm 151.47 \mathrm{sec})$. Similarly, there was no significant interaction between tests and groups $\left(\mathrm{F}_{2,10}=0.22, p=0.81 ; \eta^{2}=0.00\right)$ for $\mathrm{W}_{\max }$. There was a significant difference $\left(\mathrm{F}_{2,10}=10.65, p=0.003 ; \eta^{2}=0.12\right)$ between tests. The Pre-Test $(251.04 \pm 31.07 \mathrm{~W})$ was higher than Mid-Test $(236.46 \pm 26.41 \mathrm{~W})$ and higher than Post-Test $(229.17 \pm 29.3 \mathrm{~W})$. There was no significant difference between the MidTest and Post-Test. 
2. Relative $\mathrm{VO}_{2 \max }\left(\mathrm{ml} \cdot \mathrm{min}^{-1} \cdot \mathrm{kg}^{-1}\right)$ changes over time.

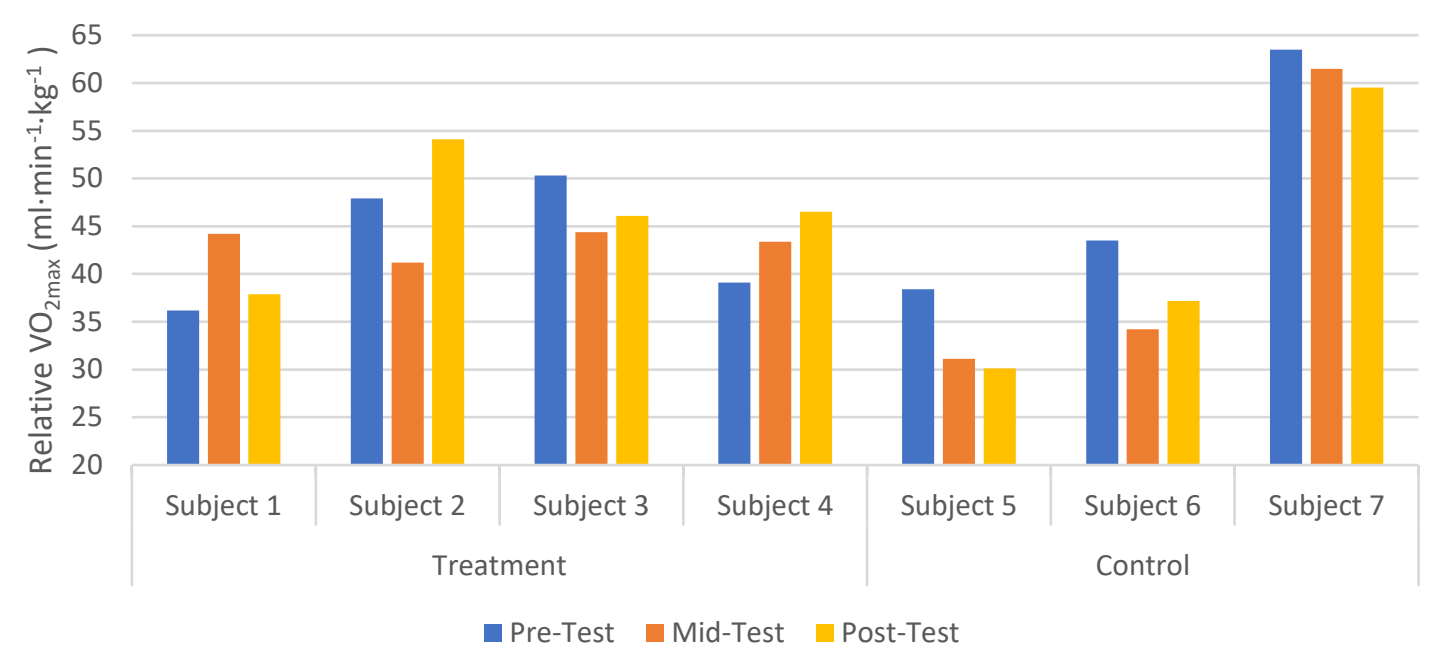

Figure 3. Time to complete $\mathrm{VO}_{2 \max }$ test

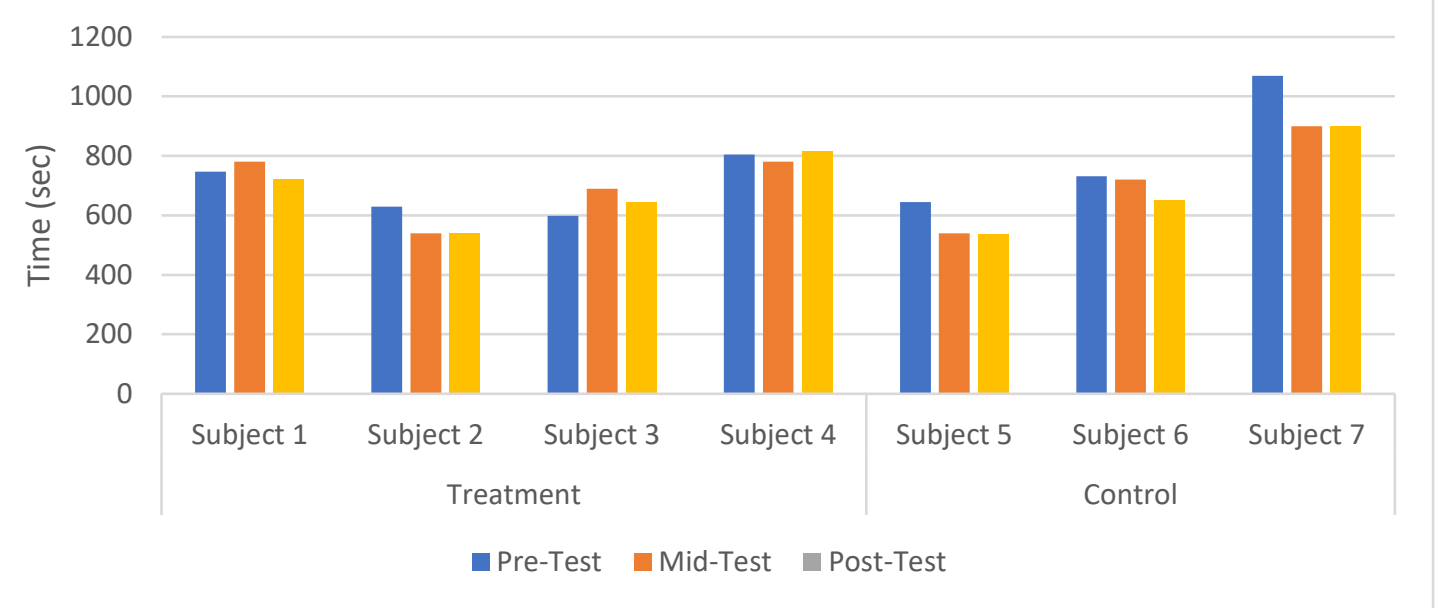

Figure 4. Maximum Watts $\left(\mathrm{W}_{\max }\right)$ performed during $\mathrm{VO}_{2 \max }$ test

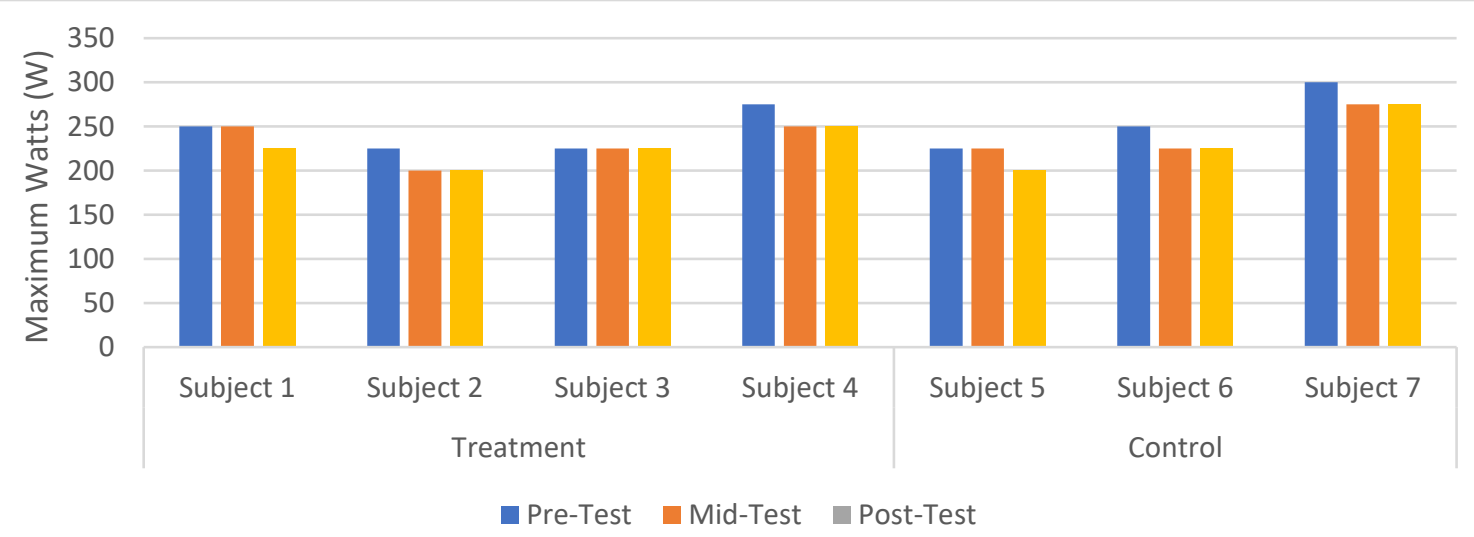




\section{Discussion}

The case series revealed that eleven 20-min training sessions using recumbent cycle ergometry at 35-45\% HRR over seven weeks with continuous BFR at $60 \%$ AOP lead to no significant differences in aerobic performance compared to a control. Yet, time to reach $\mathrm{VO}_{2 \max }$ and $\mathrm{W}_{\max }$ decreased during the duration. Previous studies have obtained aerobic adaptations from lower limb BFR with stationary cycling following a similar exercise protocol ${ }^{1,6,8}$. Oliveria et al. ${ }^{8}$ found a $5.6 \pm 4.2 \%$ increase in relative $\mathrm{VO}_{2 \max }$ over the course of six-weeks of intermittent cycle. Similarly, Abe et al. ${ }^{1}$ elicited increases in relative $\mathrm{VO}_{2 \max }$ and absolute $\mathrm{VO}_{2 \max }$ of $6.4 \%$ following 18 min of stationary cycle training one session per day, three days per week for eight weeks at $40 \%$ of $\mathrm{VO}_{2 \text { max }}$ with BFR. While this case series was similar in methodology, it did not yield similar results.

The methodology of our case series was in line with previously completed BFR training studies, aimed at increasing aerobic adaptations $1,2,6,8,9$. However, the use of stationary recumbent cycle ergometry was different. Previous studies used weight-bearing modes such as walking and traditional cycle ergometers, eliciting increases in aerobic adaptations $1,2,6,8$. These adaptations may have occurred due to the additional load placed on exercising muscle, thus augmenting BP, HR, and axial load on bones and muscles creating an increased stimulus leading to adaptations over a similar duration. The added stimulus of supporting body weight by more muscle mass recruitment and consequential oxygen consumption during traditional aerobic exercise methodology may facilitate greater stimuli for adaptation compared to non-weight bearing exercise, like recumbent cycle ergometry.

\section{Media-Friendly Summary}

Aerobic training with BFR on a recumbent cycle ergometer twice per week for seven weeks elicited no change in aerobic variables in recreationally active individuals. Practitioners seeking to use this methodology of exercise should seek further research to standardize effective training protocols accounting for appropriate intensity and duration of training session compared to upright cycling and treadmill walking.

\section{Acknowledgements}

We would like to thank the University of Lynchburg's Schewel Student-Faculty Research Fund for funding the purchase of BFR equipment.

\section{References}

1. Abe T, Fujita S, Nakajima T, Sakamaki M, Ozaki H, Ogasawara R, et al. Effects of low-intensity cycle training with restricted leg blood flow on thigh muscle volume and $\mathrm{VO}_{2 \mathrm{MAx}}$ in young men. J Sports Sci Med. 2010;1;9(3).

2. Abe T, Sakamaki M, Fujita S, Ozaki H, Sugaya M, Sato Y, Nakajima T. Effects of low-intensity walk training with restricted leg blood flow on muscle strength and aerobic capacity in older adults. J Geriatr Phys Ther $2010 \mathrm{~b} ; 33$.

3. American College of Sports Medicine. ACSM's Guidelines for Exercise Testing and Prescription $10^{\text {th }}$ ed. Wolters Kluwer. 2018.

4. Borg G.A. Psychophysical bases of perceived exertion. Med Sci Sports Exerc. 1982;14.

5. Clarkson MJ, Conway L, Warmington SA. Blood flow restriction walking and physical function in older adults: a randomized control trial. J Sci Med Sport. 2017; 20.

6. Conceicao MS, Junior EMM, Telles GD, Libardi CA, Andrade ALL, Brum PC, et al. Augmented anabolic responses after 8-wk cycling with blood flow restriction. Med Sci Sport Exerc. 2019;51(1).

7. Lambert BS, Hedt C, Moreno M, Harris JD, McCulloch P. Blood flow restriction therapy for stimulating skeletal muscle growth: practical considerations for maximizing recovery in clinical rehabilitation settings. Tech Ortho. 2018;33(2).

8. Oliveira MFM, Caputo F, Corvino RB, Denadai BS. Short-term low-intensity blood flow restricted interval training improves both aerobic fitness and muscle strength. Scand J Med Sci Sports. 2016;26(9).

9. Patterson SD, Hughes L, Warmington S, Burr J, Scott BR, Owens J, et al. Blood flow restriction exercise position stand: considerations of methodology, application, and safety. Front Physiol. 2019;10.

10. Pearson, S.J., Hussain, S.R. A Review on the Mechanisms of Blood-Flow Restriction Resistance Training-Induced Muscle Hypertrophy. Sports Med. 2015;45.

11. Warburton DER, Jamnik VK, Bredin SSD, and Gledhill N. The Physical Activity Readiness Questionnaire for Everyone (PAR-Q+) and Electronic Physical Activity Readiness Medical Examination (ePARmed-X+). Health Fit J Can.2011;4(2). 